be obtained. The results, after correction for curvature of field present in the photographs, are plotted in the accompanying graph. It must be noted that the different objectives photograph down to different sizes of particles, and this factor makes it impossible to compare the curves with each other.

It will be seen from the graph that with both the $2 \mathrm{~mm}$. and $4 \mathrm{~mm}$. objectives, when the focus is out on either side of the best focus by more than about 0.1 micron, the number of particles photographed is significantly less than the maximum number photographed. The finest particles photographed at the best focus have a thickness which is not known exactly but which is believed to be of the order of 0.06 and 0.09 microns for the two objectives respect. ively; this amount must be subtracted from the apparent depth of focus. It seems certain, therefore, that both these objectives have a depth of focus of less than 0.15 micron, and the depth of focus may actually be very much less. This could be determined more accurately by using a still finer adjustment, but, at present, time does not permit of this investigation.

\title{
Diffraction Maxima in X-Ray Photographs
}

Is a recent communication ${ }^{1} \mathrm{C}$. V. Raman and $\mathbf{P}$. Nilakantan report the presence in Laue photographs of weak intensity maxima which cannot be interpreted as Laue spots. They suggest that the new maxima are due to lattice vibrations which cause stratifications of density with the same spacing as the lattice planes, but with an orientation variable between wide limits.

The effect observed by these investigators is not new. Such weak spots are commonly found in Laue photographs taken with an $\mathrm{X}$-ray beam containing characteristic as well as continuous radiation. If the incident beam does not contain appreciable amounts of characteristic X-rays, radial streaks rather than spots appear in the Laue photographs. Earlier investigators have directed attention to these streaks or spots ${ }^{2}$, and G. D. Preston (l.c.) demonstrated that the intensity of the spots increased with the temperature. Moreover, I presented a detailed theory of the effect before the American Physical Society at its meeting on December I, 1939. An abstract of this paper was published in November last in the Bulletin of the American Physical Society and afterwards in the Physical Review ${ }^{3}$.

According to this theory, the lattice vibrations cause diffuse scattering of $\mathrm{X}$-rays showing a rapid intensity variation with the scattering direction. The intensity maxima are to be found in the directions $u_{m}$ given by the equation $(1+\lambda \tau) u_{m}=u_{0}+\lambda B_{H}$, where $u_{0}$ is the direction of incidence, $\lambda$ the wave-length of the X-rays, $B_{H}$ a reciprocal lattice vector and $\tau$ the wave vector associated with a mode of vibration of the lattice.

The assumption of Raman and Nilakantan that the new maxima are due to lattice vibrations is thus confirmed. It should be pointed out that the positions of the maxima are not given by the Bragg law as Raman and Nilakantan seem to suggest. In the Laue photograph of diamond re-

With the 8-mm. objective a certain portion of the curve is flat, but the flatness does not extend over a range of more than 0.70 microns; as the thickness of particle photographed with this objective is probably of the order of 0.14 microns, it seems that the depth of focus of this objective is certainly less than 0.55 microns and may be about 0.40 microns.

Naturally these depths of focus are increased by the adaptability of the eye when the objective is used visually.

It is obvious from these results that the present formulæ "for the depths of focus of microscope objectives give values which are considerably higher than those found experimentally.

This letter is published by kind permission of the Gold Producers Committee of the Chamber of Mines.

\section{G. BeAdLE.}

Physico-chemical Investigation Department, Transvaal Chamber of Mines, Johannesburg.

\footnotetext{
${ }^{1}$ J. Opt. Soc. America, 27, No. 11 (Nov. 1937).

${ }^{2}$ Beadle, J. Sci. Inst., 16, No. 8, 262.
} produced in their communication, the auxiliary maxima appear at a scattering angle of $43^{\circ} 0^{\prime} \pm$ $25^{\prime}$, as compared to $43^{\circ} 16^{\prime}$ deduced from the equation given above and $43^{\circ} 58^{\prime}$ from the Bragg equation.

W. H. ZaChaRIASEN.

Ryerson Physical Laboratory,

University of Chicago. May 31.

1 NATURE, 145, 667 (1940).

2 See for example, Friedrich W., Phys. $Z$. 14, 1082 (1913), Preston G. D., Proc. Roy. Soc., A, 172, 116 (1939)

${ }^{3}$ For detailed accounts see Zachariasen, W. H., Phys. Rev., 57, 597 (1940); Siegel, S., and Zachariasen, W. H., Phys. Rev., 57795 (1940).

\section{Hydration of Anilides of Normal Fatty Acids}

I HAVE previously described the formation of hydrated stearanilide ${ }^{1}$. An investigation of some of the factors influencing the binding of water by sub. stituted amides of the normal fatty acids is now in 